\title{
Effect of Constant Wall Permeability and Porous Media on the Creeping Flow through Round Vessel
}

\author{
Siddiqui $\mathrm{AM}^{1}$, Siddiqa $\mathrm{S}^{2}$ and Naqvi $\mathrm{AS}^{3 *}$ \\ ${ }^{1}$ Department of Mathematics, Pennsylvania State University, York Campus, Old Main, State College, USA \\ ${ }^{2}$ Department of Mathematics, COMSATS Institute of Information Technology, Islamabad, Pakistan
}

${ }^{3}$ Department of Electrical Engineering, COMSATS Institute of Information Technology, Sahiwal, Pakistan

\begin{abstract}
The present study reports the influence of constant wall permeability and porous media on the creeping flow passing through round vessel. The inverse method is used to obtain the exact solution. To understand the behaviour of flow, the mathematical expression for stream function, velocity components, wall shear stress, pressure distribution, flow rate and leakage flux are provided. Our main purpose here is to demonstrate the constant permeability of the wall and of porous media on the existence of flow properties because of its applicability "flow through permeable renal disease tubule" in biological sciences. Flow properties at an entrance, mid place and at exit are discussed graphically for involved parameters and it is observed that low permeability of porous medium slow down the velocity of fluid along axial coordinate while minor change is observed along radial coordinate. While constant wall permeability shows the opposite behaviour on the same properties. Fractional permeation rate from the diseased vessel, wall permeation velocity, pressure drop and leakage flow rate are tabulated for physiological data of rat kidney. It is observed that for increasing wall permeability and high osmotic pressure drop provided maximum fractional permeation rate $84 \%$. It is also noted that leakage flow and the amount of permeate fluid are independent of porosity of porous media, they only depend on the constant wall permeability. Comparison of the present study with the previous work in view of fractional permeation rate is also provided in the results section.
\end{abstract}

Keywords: Constant wall permeation; Porous media; Diseased renal tubule; Fractional permeation rate (FPR); Partial obstruction

\section{Introduction}

In past years, flow through porous media regime has been a subject of intensive study in a field of sciences with varying degree of success due to its important application in engineering, industry and medical fields. Production of petroleum and natural gasses, well drilling, lodging, fluid flow through obstructed renal tubules require many predictions based on results of fluid flow through a porous media. The motion of the fluid is affected by so many factors. The boundaries of the fluid affect the flow to have stationery boundaries, fluctuating boundaries, moving boundaries, oscillatory boundaries, porous boundaries and so on. The fluid motion in ducts, parallel plate channels, rectangular channels, parabolic boundaries, circular and cylindrical boundaries have been studied due to their natural existence.

There are many flows (gastric and circulatory) through which garbage or waste material may be deposited in the vessels (arteries, veins, renal tubules, capillaries, and intestine) and it leads to a diseased system $[1,2]$. Therefore, the normal vessel that contains porous media can be treated using the model which evaluates supplementary drag forces exerted on the flow due to the presence of solid matrix numbers emphasized by Khalid and Vafai [3]. These matrix numbers may be some kind of food item which cannot be digested, bacterial masses or fatty bunch. The viscosity of such materials will also be a cause of disturbance to the flow through the circular tube. Some numbers may causes of fully blockage and some partially. In partial blockage, some material allowed to pass through the blocked zone and this effectiveness can be a deal, modeled and analyzed as porous media regime. When the fatty and fibrous tissues are clotted in the wall lumen, its distribution acts like a porous medium. The general equation of motion for the flow of a viscous fluid through a porous media has been derived by Ahmadi and Manvi [4]. The porous material containing the fluid is, in fact, a non-homogenous media. For the sake of analysis, it is possible to replace it with a homogenous fluid which has dynamic properties equivalent to the local averages of the original non-homogenous medium.

Homogenous fluid flow through the circular vessel with constant and variable permeable wall has its own status and proposed by many authors [5-20] in past and most recent years with a special application of flow through the kidney. Kidneys are most important re-absorption and filtration plant in the body. Each kidney contains millions of nephrons. Nephrons are functional unit of the kidney. They consist of the glomerulus and renal tubules which are originating from the tuft of the glomerulus. Renal tubules are involved in one of the most important and final stages of the nephron function: Clearing the end products of metabolism and maintaining the volume of the body fluids. The major portion of the tubular function is being carried out by the proximal renal tubule. The tubular epithelium is highly permeable to water and small solutes, to facilitate their re-absorption from glomerular filtrate. It is generally accepted that fluid is transported across the permeable wall by trans-boundary pressure drop (TPD) which includes osmotic pressure due to proteins and other solutes. Thus, it is expected that the hydrodynamic of renal flow play a significant role in understanding the nephron function. The proximal renal tubules are not uniform all along their length and the wall permeability may also vary in

*Corresponding author: Naqvi S, Department of Electrical Engineering COMSATS Institute of Information Technology, Sahiwal, Pakistan, Tel: (92-51) 9247000-3; E-mail: naqvi.sehrish@yahoo.com

Received May 02, 2018; Accepted May 29, 2018; Published June 10, 2018

Citation: Siddiqui AM, Siddiqa S, Naqvi AS (2018) Effect of Constant Wall Permeability and Porous Media on the Creeping Flow through Round Vessel. J Appl Computat Math 7: 399. doi: 10.4172/2168-9679.1000399

Copyright: (c) 2018 Siddiqui AM. This is an open-access article distributed under the terms of the Creative Commons Attribution License, which permits unrestricted use, distribution, and reproduction in any medium, provided the original author and source are credited. 
different portions of the tube. It is, therefore, appropriate to consider a theoretical model for renal flow with non-uniform tube cross-section and variable wall permeability.

This work is the influence of past research on the flow through the healthy vessel, the affected (diseased) vessel is under consideration. The motive of this work is to extend the work [15] by taking round vessel with effects of porous media and constant wall permeability. Our model is similar to the renal tubule, therefore all results in the present work are related to this application along with physiological data of rat kidney. The axisymmetric coordinate is chosen according to the geometry of the problem. The hydrodynamics equations for creeping flow of viscous fluid passing through the porous media within the permeable vessel are transformed. Exact solutions are obtained with the help of inverse method [21-27]. Ana-lytic expressions are derived for axial and radial velocities, flow rate, pressure distribution, pressure drop, wall shear stress and leakage flux. Effects of porous media, wall permeability and entrance flow rate on the flow characteristics are visualized graphically. FPR \%, wall permeability rate, pressure drop, leakage flux and comparison of the present study with the published work are tabulated in the result section. In the end, the detailed conclusion of this study is also provided.

\section{Problem Formulation}

Creeping flow of an incompressible viscous fluid passing through round vessel filled with porous media has been considered. Length and radius of the vessel are $\mathrm{L}$ and $\mathrm{R}$. The flow dynamics are about axial and radial direction, therefore, the axisymmetric coordinates $(\mathrm{r} ; 0 ; \mathrm{z})$ are selected according to the geometry of the problem in which axial axis is along the axis of the vessel and radial axis is perpendicular to it. The wall re-absorption is assumed to be constant. The radial component of velocity given by $\mathrm{v}_{\mathrm{r}}(\mathrm{r} ; \mathrm{z})$ is due to the constant re-absorption at the wall and axial component $\mathrm{v}_{\mathrm{z}}(\mathrm{r} ; \mathrm{z})$ is an extension of the vessel along which the flow is symmetric. The governing equations for such flow are given by the following set of equations:

$$
\begin{aligned}
& \mathrm{v}=\left[\mathrm{v}_{\mathrm{r}}(\gamma ; \mathrm{z}) ; 0 ; \mathrm{v}_{\mathrm{z}}(\gamma ; \mathrm{z})\right. \\
& \frac{1}{\gamma} \frac{\partial}{\partial \gamma}\left(\gamma v_{\gamma}\right)+\frac{\partial v_{z}}{\partial z}=0 \\
& \frac{\partial p}{\partial \gamma}=\mu\left(\frac{\partial^{2} v_{\gamma}}{\partial \gamma^{2}}+\frac{1}{\gamma} \frac{\partial v_{\gamma}}{\partial \gamma}-\frac{v_{\gamma}}{\gamma^{2}}+\frac{\partial^{2} v_{z}}{\partial z^{2}}\right)-\frac{\mu}{k} v_{\gamma} \\
& \frac{\partial p}{\partial \gamma}=\mu\left(\frac{\partial^{2} v z}{\partial \gamma^{2}}+\frac{1}{\gamma} \frac{\partial v_{z}}{\partial \gamma}+\frac{\partial^{2} v_{z}}{\partial z^{2}}\right)-\frac{\mu}{k} v_{z}
\end{aligned}
$$

where $\mathrm{p}(\gamma ; \mathrm{z}), \mu, \mathrm{k}, \mathrm{v}_{\mathrm{r}} ; \mathrm{v}_{\mathrm{z}}, \gamma, \mathrm{z}$ are the hydrostatic fluid pressure inside the vessel, the coefficient of viscosity, the permeability parameter, the velocity along radial direction, the velocity along axial direction, the radial coordinate, and the axial coordinate respectively. The appropriate boundary conditions of the problem are:

$$
\begin{aligned}
& v_{\gamma}=0, \frac{\partial v_{z}}{\partial \gamma}=0 \text { at } \gamma=0 \\
& v_{\gamma}=v_{0}, v_{z}=0 \text { at } \gamma=R \\
& 2 \pi \int_{0}^{R} \lambda v_{z}(\gamma, \mathrm{z}) d \gamma=Q_{0} \text { at } z=0, \\
& \mathrm{p}=\mathrm{p}_{0} \text { at } \mathrm{z}=0 \text { and } \mathrm{p}=\mathrm{pl} \text { at } \mathrm{z}=\mathrm{L}
\end{aligned}
$$

where $\mathrm{v}_{\mathrm{o}}$ wall permeability, $\mathrm{Q}_{\mathrm{o}}$ the entrance flow rate, $\mathrm{p}_{0}$ the pressure at entrance and $\mathrm{p}_{\mathrm{L}}$ the pressure at exit of vessel (Figure 1).

By elimination of pressure from eqn.(3) and eqn.(4); we arrived at:

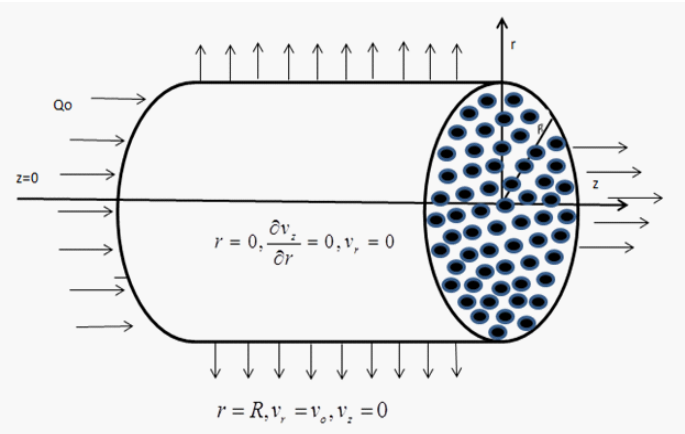

Figure 1: Schematic evolution of slow flow through porous vessel.

$$
\frac{\partial}{\partial \gamma}\left\{\frac{1}{\gamma} \frac{\partial}{\partial \gamma}\left(\gamma \frac{\partial v_{z}}{\partial_{\gamma}}\right)\right\}+\frac{\partial^{3} v_{z}}{\partial \gamma \partial z^{2}}-\frac{\partial}{\partial z}\left\{\frac{\partial}{\partial \gamma}\left(\frac{1}{\gamma} \frac{\partial}{\partial \gamma}\left(\gamma v_{\gamma}\right)\right)\right\}-\frac{\partial^{3} v_{\gamma}}{\partial z^{3}}+\frac{1}{k}\left(\frac{\partial v_{\gamma}}{\partial z}-\frac{\partial v_{z}}{\partial \gamma}\right)=0
$$

taking partial derivative of eqn.(7) with respect to $\mathrm{z}$ and using eqn.(2), we obtained:

$$
\begin{aligned}
& \frac{\partial}{\partial z}\left[\frac{1}{\gamma} \frac{\partial}{\partial \gamma}\left\{\gamma \frac{\partial}{\partial \gamma}\left(\frac{1}{\gamma} \frac{\partial}{\partial \gamma}\right)\right\}\right]\left(\gamma v_{z}\right)+2 \frac{\partial}{\partial \gamma}\left\{\frac{1}{\gamma} \frac{\partial}{\partial \gamma}\left(\frac{\partial^{2}}{\partial z^{2}}\right)\right\}\left(\gamma v_{\gamma}\right)+\frac{1}{\gamma} \frac{\partial^{4}}{\partial z^{4}}\left(\gamma v_{\gamma}\right)+ \\
& \quad+\frac{1}{k}\left(\left\{\frac{\partial}{\partial \gamma}\left(\frac{1}{\gamma} \frac{\partial}{\partial_{\gamma}}\right)\right\}+\frac{1}{\gamma} \frac{\partial^{2}}{\partial z^{2}}\right)\left(\gamma v_{\gamma}\right)=0
\end{aligned}
$$

The velocity components in term of stream function $\psi(\gamma ; z)$ are defined by:

$$
v_{\gamma}=\frac{1}{\gamma} \frac{\partial \psi}{\partial z}, v_{z}=-\frac{1}{\gamma} \frac{\partial \psi}{\partial \gamma}
$$

Using eqn. (9), the eqn. (8) is finally reduces to the fourth order expression:

$$
E^{4} \psi-\frac{1}{k} E^{2} \psi=0
$$

or it can be re-written as:

$$
E^{2}\left(E^{2} \psi\right)-\frac{1}{k}\left(E^{2} \psi\right)=0
$$

where the operator $\mathrm{E}^{2}$ is defined as:

$$
E^{2}=\frac{\partial^{2}}{\partial \gamma^{2}}-\frac{1}{\gamma} \frac{\partial}{\partial \gamma}+\frac{\partial^{2}}{\partial Z^{2}}
$$

The transformed boundary conditions in term of stream function are:

$$
\begin{aligned}
& \frac{\partial \psi}{\partial z}=0, \frac{-1}{\gamma} \frac{\partial^{2} \psi}{\partial \gamma^{2}}+\frac{1}{\gamma^{2}} \frac{\partial \psi}{\partial \gamma}=0 \text { at } \gamma=0, \\
& \frac{\partial \psi}{\partial z}=v_{0} \gamma, \frac{\partial \psi}{\partial \gamma}=0 \text { at } \gamma=R \\
& -\frac{Q_{0}}{2 \pi}=\psi(R, z)-\psi(0, z) \text { at } z=0
\end{aligned}
$$

typically $\psi(0 ; 0)=0$ so that eqn. (14) becomes:

$$
-\frac{Q_{0}}{2 \pi}=\psi(R, z) \text { at } z=0
$$

We finally obtain the bi-harmonic equation eqn. (11) along with non-homogenous boundary conditions eqns. (12-15), presenting creeping flow through the porous vessel with constant wall permeability.

\section{Problem Solution}

By proposing stream function of the form: 
$\psi(\gamma ; \mathrm{z})=\mathrm{v}_{\mathrm{o}} \mathrm{z} f(\gamma)+\mathrm{g}(\gamma)$

where $f(\gamma)$ and $g(\gamma)$ are arbitrary functions. Using above assumption in eqns. (11-15); the following two differential equations in term of arbitrary functions are retrieved:

$$
\left(\frac{\partial^{2}}{\partial \gamma^{2}}-\frac{1}{\gamma} \frac{d}{d \gamma}\right)\left\{\left\{\left(\frac{d^{2}}{d \gamma^{2}}-\frac{1}{\gamma} \frac{d}{d \gamma}\right)-\frac{1}{k}\right\} f(\gamma)=0\left(\frac{d^{2}}{d \gamma^{2}}-\frac{1}{\gamma} \frac{d}{d \gamma}\right)\left\{\left(\frac{d^{2}}{d \gamma^{2}}-\frac{1}{\gamma} \frac{d}{d \gamma}\right)-\frac{1}{k}\right\} g(\gamma)=0\right.
$$

Associated boundary conditions are:

$$
\begin{aligned}
& \frac{f(\gamma)}{\gamma}=0, \frac{d}{d \gamma}\left(\frac{1}{\gamma} \frac{d}{d \gamma}\right) f(\gamma)=0, \frac{d}{d \gamma}\left(\frac{1}{\gamma} \frac{d}{d \gamma}\right) g(\gamma)=0 \text { at } \gamma=0 \\
& \frac{f(\gamma)}{\gamma}=1, \frac{d f(\gamma)}{d \gamma}=0, \frac{d g(\gamma)}{d \gamma}=0 \text { at } \gamma=R \\
& g(R)-g(0)=\frac{-Q_{0}}{2 \pi} \text { at } z=0
\end{aligned}
$$

The solution of Eq. (17) is:

$$
f(\gamma)=\frac{2 \gamma I_{1}\left(\frac{\gamma}{\sqrt{k}}\right)-\frac{\gamma^{2}}{\sqrt{k}} I_{0}\left(\frac{R}{\sqrt{k}}\right)}{2 I_{1}\left(\frac{R}{\sqrt{k}}\right)-\frac{R}{\sqrt{k}} I_{0}\left(\frac{R}{\sqrt{k}}\right)} \text { and } g(\gamma)=-\frac{Q_{0}}{2 \pi R} \frac{2 \gamma I_{1}\left(\frac{\gamma}{\sqrt{k}}\right)-\frac{\gamma^{2}}{\sqrt{k}} I_{0}\left(\frac{R}{\sqrt{k}}\right)}{2 \pi R 2 I_{1}\left(\frac{R}{\sqrt{k}}\right)-\frac{R}{\sqrt{k}} I_{0}\left(\frac{R}{\sqrt{k}}\right)}
$$

where $I_{1} ; I_{2}$ are modified bessel functions [21] of order 1 and 2: The expression for stream function is obtained as:

$$
\psi(\gamma, z)=v_{0} \frac{2 \gamma I_{1}\left(\frac{\gamma}{\sqrt{k}}\right)-\frac{\gamma^{2}}{\sqrt{k}} I_{0}\left(\frac{R}{\sqrt{k}}\right)}{2 I_{1}\left(\frac{R}{\sqrt{k}}\right)-\frac{R}{\sqrt{k}} I_{0}\left(\frac{R}{\sqrt{k}}\right)}-\frac{Q_{0}}{2 \pi R} \frac{2 \gamma I_{1}\left(\frac{\gamma}{\sqrt{k}}\right)-\frac{\gamma^{2}}{\sqrt{k}} I_{0}\left(\frac{R}{\sqrt{k}}\right)}{2 I_{1}\left(\frac{R}{\sqrt{k}}\right)-\left(\frac{R}{\sqrt{k}}\right) I_{0}\left(\frac{R}{\sqrt{k}}\right)}
$$

both radial and axial velocity components are respectively obtained as:

$$
v_{\gamma}(\gamma, z)=v_{0} \frac{2 I_{1}\left(\frac{\gamma}{\sqrt{k}}\right)-\left(\frac{\gamma}{\sqrt{k}}\right) I_{0}\left(\frac{R}{\sqrt{k}}\right)}{2 I_{1}\left(\frac{R}{\sqrt{k}}\right)-\frac{R}{\sqrt{k}} I_{0}\left(\frac{R}{\sqrt{k}}\right)} \text { and } v_{z}(\gamma, z)=-\frac{\frac{1}{\sqrt{k}} I_{0}\left(\frac{\gamma}{\sqrt{k}}\right)-\frac{1}{\sqrt{k}} I_{0}\left(\frac{R}{\sqrt{k}}\right)}{2 I_{1}\left(\frac{R}{\sqrt{k}}\right)-\frac{R}{\sqrt{k}} I_{0}\left(\frac{R}{\sqrt{k}}\right)}\left(2 v_{0} z-\frac{Q_{0}}{\pi R}\right)
$$

The longitudinal (axial) flow rate denoted by $\mathrm{Q}(\mathrm{z})$ and leakage ow rate after straight forward computation are given as respectively:

$$
\begin{aligned}
& Q(z)=\int_{0}^{R} 2 \pi \gamma v_{z}(\gamma, 0) d r=Q_{0}-2 \pi R v_{0} z \\
& q(z)=\frac{d Q(z)}{d z}=2 \pi R v_{0}
\end{aligned}
$$

The expression for pressure distribution in the vessel by the cross integration of eqn.(3) and eqn. (4) yields the solution for $\mathrm{p}(\gamma ; \mathrm{z})$ in term of $\mathrm{r}$ and $\mathrm{z}$; the average pressure of fluid at end point, pressure drop over the length $\mathrm{L}$ of the leaky vessel and wall shear stress at wall are respectively given below:

$$
\begin{aligned}
& \frac{1}{\mu} p(\gamma, z)=2 v_{0} \frac{\frac{1}{\sqrt{k}} I_{0}\left(\frac{\gamma}{\sqrt{k}}\right)-\frac{1}{\sqrt{k}} I_{0}\left(\frac{R}{\sqrt{k}}\right)}{2 I_{1}\left(\frac{R}{\sqrt{k}}\right)-\frac{R}{\sqrt{k}} I_{0}\left(\frac{R}{\sqrt{k}}\right)}+\left(v_{0} z-\frac{Q_{0}}{\pi R}\right) \frac{\frac{z}{(k)^{3 / 2}} I_{0}\left(\frac{\gamma}{\sqrt{k}}\right)}{2 I_{1}\left(\frac{R}{\sqrt{k}}\right)-\frac{R}{\sqrt{k}} I_{0}\left(\frac{R}{\sqrt{k}}\right)}+\frac{p_{0}}{\mu} \\
& \bar{p}(z)=\frac{\int_{0}^{R} p(\gamma, z) 2 \pi \gamma d \gamma}{\int_{0}^{R} 2 \pi \gamma d \gamma}=\frac{2 v_{0} \mu}{R}+\frac{2 \mu z}{k R^{2}}\left(v_{0} z-\frac{Q_{0}}{\pi R}\right) \frac{I_{1}\left(\frac{R}{\sqrt{k}}\right)}{2 I_{1}\left(\frac{R}{\sqrt{k}}\right)-\frac{R}{\sqrt{k}} I_{0}\left(\frac{R}{\sqrt{k}}\right)} \\
& \Delta \bar{p}=\bar{p}(0)-\bar{p}(L)=-\frac{2 \mu L}{k R^{2}}\left(v_{0} L-\frac{Q_{0}}{\pi R}\right) \frac{I_{1}\left(\frac{R}{\sqrt{k}}\right)}{2 I_{1}\left(\frac{R}{\sqrt{k}}\right)-\frac{R}{\sqrt{k}} I_{0}\left(\frac{R}{\sqrt{k}}\right)}
\end{aligned}
$$

$$
\tau_{\gamma}=R=-\mu\left(\frac{\partial v_{z}}{\partial \gamma}+\frac{\partial v_{\gamma}}{\partial_{z}}\right)=-\frac{{ }^{\frac{\mu}{k}} I_{1}\left(\frac{R}{\sqrt{k}}\right)}{2 I_{1}\left(\frac{R}{\sqrt{k}}\right)-\frac{R}{\sqrt{k}} I_{0}\left(\frac{R}{\sqrt{k}}\right)}\left(2 v_{0} z "-\frac{Q_{0}}{\pi R}\right)
$$

The amount of permeate fluid from the wall of circular vessel can be defined as fractional permeation rate [15]. It can be computed as:

$$
F P R=\frac{Q(0)-Q(L)}{Q(0)}=\frac{2 \pi R v_{0} L}{Q_{0}}
$$

which shows the dependence of $\mathrm{F} P \mathrm{R}$ on constant wall permeability $\mathrm{v}_{\mathrm{o}}$ (re-absorption velocity) and axial flow rate $\mathrm{Q}_{\mathrm{o}}$.

\section{Results and Discussion}

In this section, results are analysed in two ways; one is the theoretical results which are generated by Mathematica for varying one flow parameter and fixing others, and other is physiological results i.e., done for all relevant values of physiological situation. Introducing nondimensionalization for the convenience of the graphical representation:

$$
\gamma^{*}=\frac{\gamma}{R}, z^{*}=\frac{z}{R}, v^{*}=\frac{v}{U}, p^{*}=\frac{p}{\mu U / R}, k^{*}=\frac{k}{R^{2}}
$$

where $\mathrm{U}$ is the fluid entrance velocity. All the results are predicted along different axial positions namely; Entrance $(\mathrm{z}=0: 1)$, middle $(\mathrm{z}=0: 3)$ and exit of the vessel $(\mathrm{z}=0: 6)$ for the length of vessel equals to 0:67. In Figure 2 , radial velocity increases for increasing permeability parameter of porous media but remains symmetric at all position downstream due to constant permeation. Figure 3 depicted the increasing behavior of

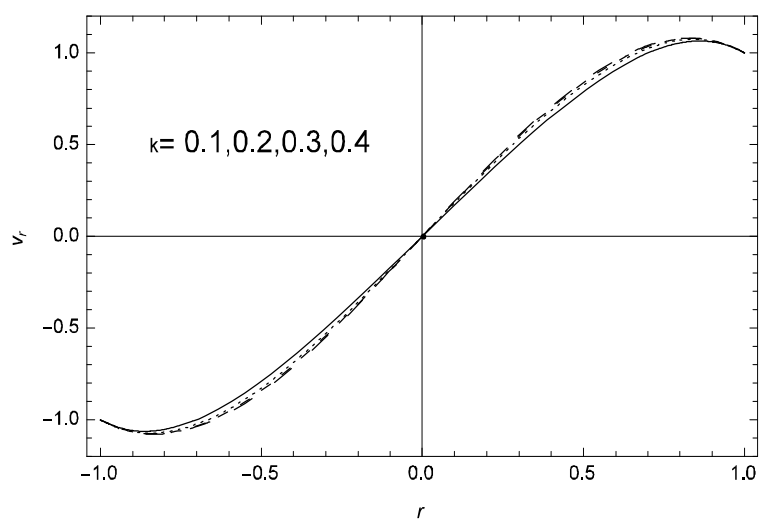

Figure 2: Effect of $k$ on $v_{r}$.

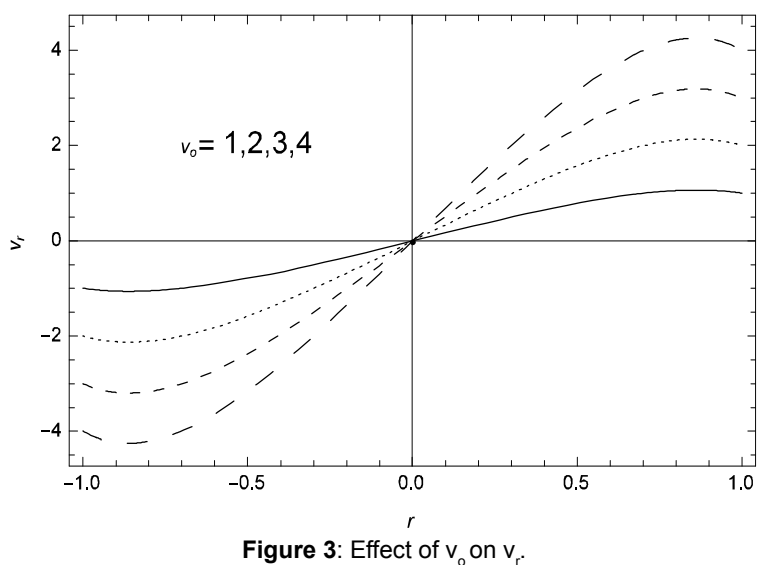


radial velocity for increasing rate of wall permeation velocity $\mathrm{v}_{0}$. Figures 4-6 is plotted against the increasing values of permeability $\mathrm{k}$ of porous media on the axial velocity at the entrance, mid place and exit of the vessel. It is observed that axial velocity gradually increases when the permeability $\mathrm{k}$ increases. Axial velocity decreases for increasing wall permeation velocity $v_{0}$, (Figures 7-9). From Figures 10 and 11 showed that vessel hydrostatic pressure distribution increases linearly for increasing $\mathrm{k}$ and $\mathrm{v}_{\mathrm{o}}$.

For theoretical and experimental point of view, physiological data of rat kidney is given in Table 1. Variation in F P R for different
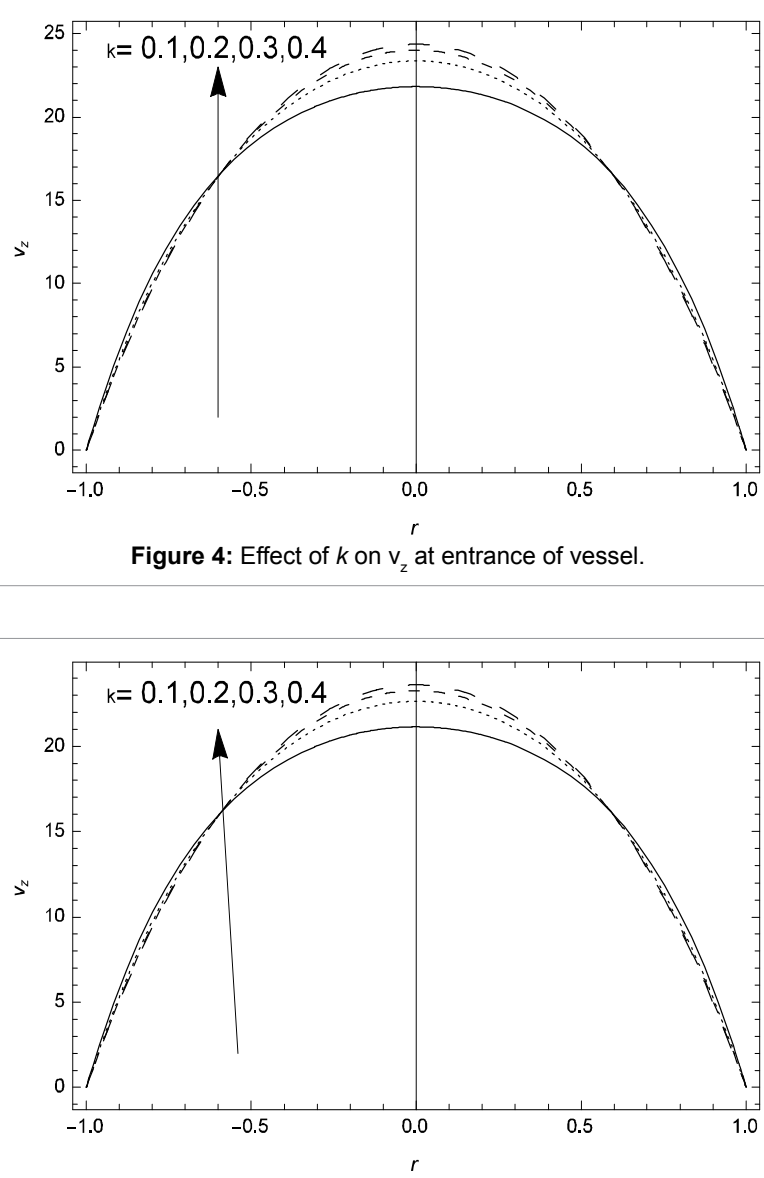

Figure 5: Effect of $k$ on $v_{z}$ at mid place of vessel.

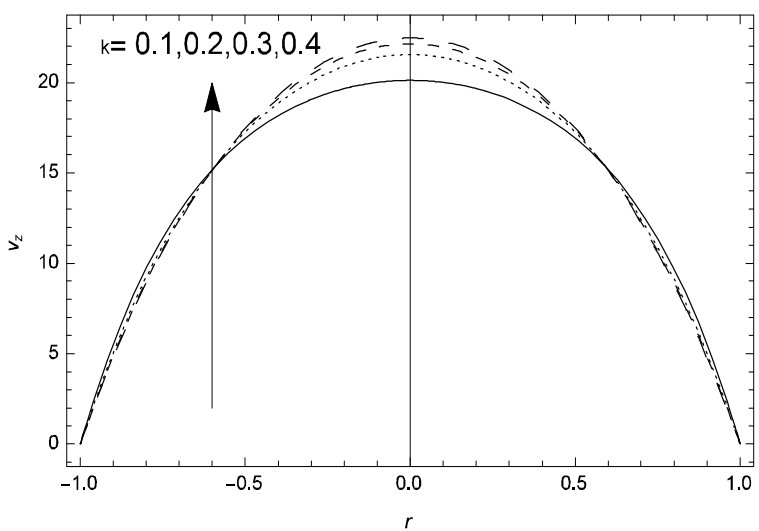

Figure 6: Effect of $k$ on $v_{z}$ at exit of vessel.

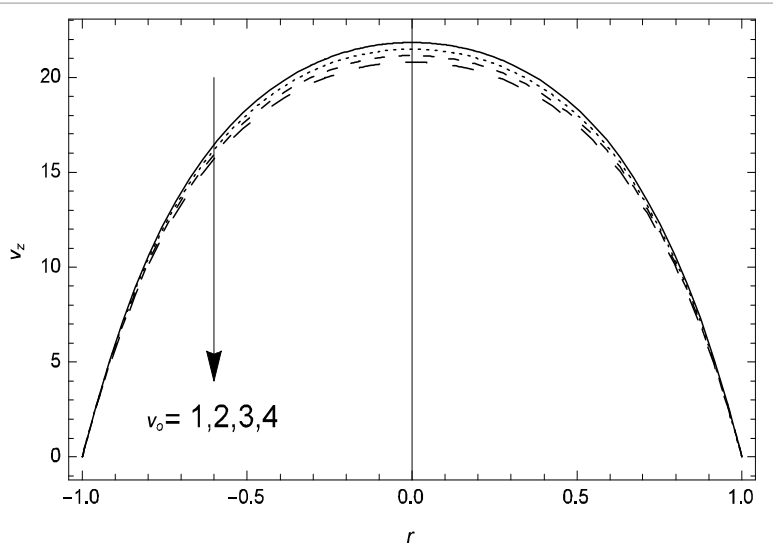

Figure 7: Effect of $v_{0}$ on $v_{z}$ at entrance of vessel.

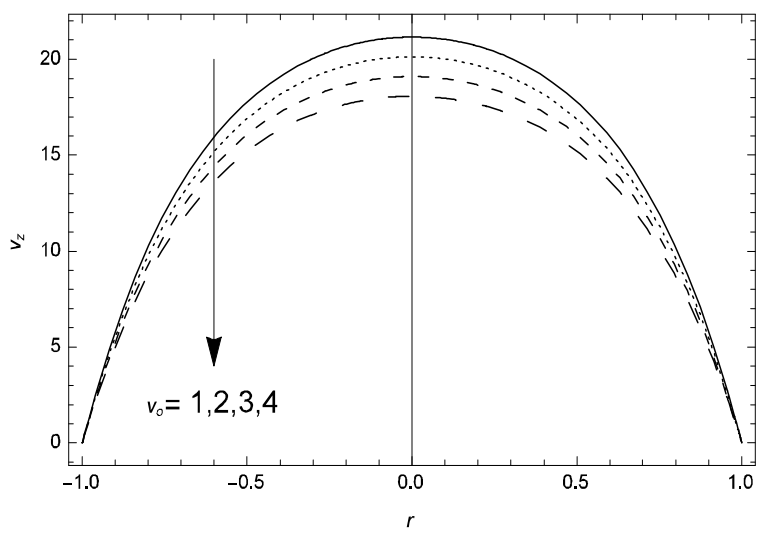

Figure 8: Effect of $v_{o}$ on $v_{z}$ at mid place of vessel.

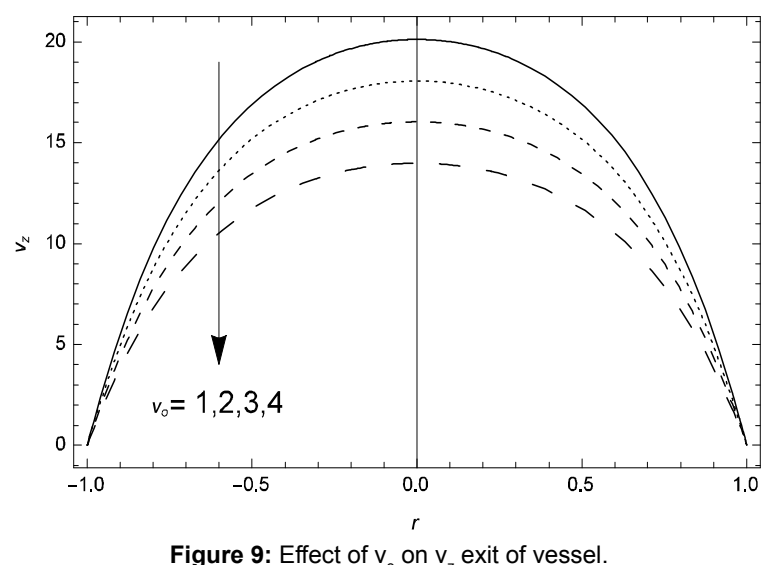

values of wall permeability, pressure drop, and leakage flux has been given in Table 2 with the help of physiological data provided in Table 1. It is observed that for increasing wall permeability, pressure drop and leakage flow rate the F P R through walls attained its maximum value for the case of the constant rate of permeation at the wall. Table 3 shows the comparison of F P R\% with previous work [15]. It is observed that the percentage of fractional permeation rate difference with experimental data of rat kidney is higher in diseased vessel than the healthy vessel. 
Citation: Siddiqui AM, Siddiqa S, Naqvi AS (2018) Effect of Constant Wall Permeability and Porous Media on the Creeping Flow through Round Vessel. J Appl Computat Math 7: 399. doi: 10.4172/2168-9679.1000399

Page 5 of 6

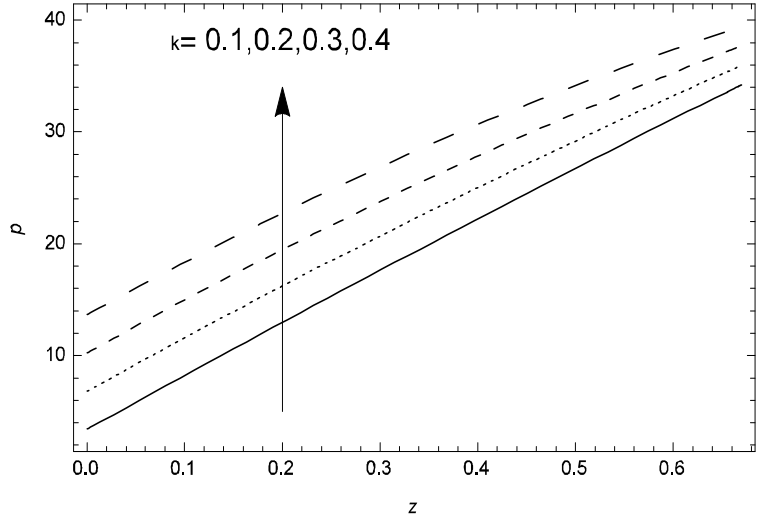

Figure 10: Effect of $k$ on $p$.

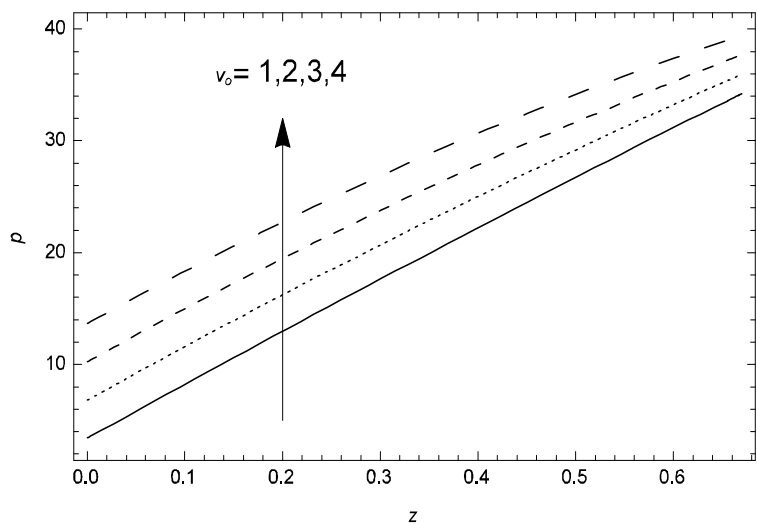

Figure 11: Effect of $v_{0}$ on $p$

\begin{tabular}{|l|c|c|c|}
\hline Quantity & Symbols & Values & Units \\
\hline Radius tube & $\mathrm{R}$ & 0.00108 & $\mathrm{Cm}$ \\
\hline Length Tube & $\mathrm{L}$ & 0.67 & $\mathrm{Cm}$ \\
\hline $\begin{array}{l}\text { Entrance } \\
\text { Pressure }\end{array}$ & $\mathrm{P}_{\mathrm{c}}$ & 14400. & $\frac{\mathrm{dYn}}{\mathrm{cm}^{2}}$ \\
\hline $\begin{array}{l}\text { Entrance Flow } \\
\text { Rate }\end{array}$ & $\mathrm{Q}_{\mathrm{O}}$ & $4.08 \times 10^{-8}$ & $\frac{\mathrm{cm}^{2}}{\mathrm{sec}}$ \\
\hline Viscosity & $\mu$ & 0.00737 & $\frac{d Y n \cdot \mathrm{sec}}{\mathrm{cm}}$ \\
\hline
\end{tabular}

Table 1: Physiological data for the rate kidney.

\begin{tabular}{|c|c|c|c|}
\hline FAR\% & Wall permeability*10-4 & Pressure drop ${ }^{*} 10^{6}$ & Leakage flow rate $* 10^{-6}$ \\
\hline 84 & 1.6 & -4.29842 & 1.08573 \\
\hline 78 & 1.5 & -4.49296 & 1.01788 \\
\hline 73 & 1.4 & -4.6875 & 0.95002 \\
\hline 68 & 1.3 & -4.88204 & 0.88216 \\
\hline
\end{tabular}

Table 2: Variation of fractional permeation rate FPR, for different values of wall permeation velocity, pressure drop and leakage flow rate.

\begin{tabular}{|c|c|c|c|c|}
\hline FPR\% & $\begin{array}{c}\text { FPR difference with } \\
\text { experimental data\% }\end{array}$ & Flow & $\begin{array}{c}\text { Assumed } \\
\text { Geometry }\end{array}$ & $\begin{array}{c}\text { Status of } \\
\text { renal tubule }\end{array}$ \\
\hline 80 & 7 & $\begin{array}{c}\text { Homogenous } \\
\text { regime }\end{array}$ & Parallel plates & healthy \\
\hline 84 & 11 & $\begin{array}{c}\text { Medium } \\
\text { porous regime }\end{array}$ & Round vessel & Diseased \\
\hline
\end{tabular}

Table 3: FPR\% difference with experimental value that is $73 \%$ for the constant wall permeation $\mathrm{v}_{\mathrm{o}}=1: 6$.

\section{Conclusion}

Here we have supposed wall permeability of round vessel as a constant function of axial distance. The vessel is filled with some waste material which is playing a role of porous media. Results are interpreted against the effect of porous media and constant wall permeation. Physiological data of rat kidney is used to interpret the results. Important conclusions are as followed: When the porosity of media is higher, then fluid will stay longer in the vessel and hence the percentage of FPR will increases (that is $84 \%$ ) and its difference with actual experimental results is $11 \%$. This study stated the swear condition of the diseased permeable vessel (leaky diseased renal tubule). The previous findings can be obtained by setting: permeability parameter (k) of porous media approaches to infinity, we can obtain the solution of the homogenous fluid regime with constant wall permeability [9]. It is also observed that if wall permeability $\left(\mathrm{v}_{\mathrm{o}}\right)$ approaches to zero and parameter (k) approaches to infinity then classical results of Poiseuille flow (homogenous fluid regime with impermeable boundary) can be achieved. If $\mathrm{v}_{\mathrm{o}}$ approaches to zero but $\mathrm{k}$ is set to be free then flow through porousmediaregime with a homogenous wallisretrieved where nopermeation has been taken from the walls of the vessel. Since constant wall permeability of vessel is not the ideal case, thus the present model may be improved by taking appropriate variable wall permeability of round vessel filled with some partial obstruction.

\section{References}

1. Kulaylat MN, Doerr RJ (2001) Small bowel obstruction. Surgical Treatment: Evidence-Based and Problem-Oriented.

2. Takeuchi K, Satoh H (2010) Measurement of small intestinal damage. Current protocols in toxicology, pp: 21-7.

3. Khaled AR, Vafai K (2003) The role of porous media in modelling flow and heat transfer in biological tissues. Int J Heat Mass Transfer 46: 4989-5003.

4. AhmadiG, Manvi R (1971) Equation of motion for viscous flow through a rigid porous medium. Indian J Technol 9: 441.

5. LG Wesson JR (1954) A theoretical analysis of urea excretion by the mammalian kidney. Am J Physiol 17: 364-371.

6. Burgen ASV (1956) A theoretical treatment of glucose reabsorption in the kidney.Can J Biochem Physiol 34: 466-474.

7. Macey RI (1963) Pressure flow patterns in a cylinder with reabsorbing walls The Bulletin of Mathematical Biophysics 25: 1-9.

8. Kelman RB (1962) A theoretical note on exponential flow in the proximal part of the mammalian nephron. The Bulletin of Mathematical Biophysics 24: 303-317.

9. Macey RI (1965) Hydrodynamics in the renal tubule. The Bulletin of Mathematical Biophysics 27: 117

10. Kozinski AA, Schmidt FP, Lightfoot EN (1970) Velocity profiles in porous-walled ducts. Industrial \& Engineering Chemistry Fundamentals 9: 502-505.

11. Marshall EA, Trowbridge EA (1974) Flow of a Newtonian fluid through a permeable tube: the application to the proximal renal tubule. Bulletin of Mathematical Biology 36: 457-476.

12. Palatt PJ, Sackin H, Tanner RI (1974) A hydrodynamic model of a permeable tubule. J Theor Biol 44: 287-303.

13. Chaturani P, Ranganatha TR (1991) Flow of Newtonian fluid in non-uniform tubes with variable wall permeability with application to flow in renal tubules. Acta Mechanica 88: 11-26.

14. Radhakrishnamacharya G, Chandra P, Kaimal MR (1981) A hydro dynamical study of the flow in renal tubules. Bulletin of Mathematical Biology 43: 151-163.

15. Haroon T, Siddiqui AM, Shahzad A (2016) Creeping flow of viscous fluid through a proximal tubule with uniform reabsorption: a mathematical study. App Math Sci 10: 795-807.

16. Kapur JN (1985) Mathematical models in biology and medicine. Affiliated EastWest Press. 
Citation: Siddiqui AM, Siddiqa S, Naqvi AS (2018) Effect of Constant Wall Permeability and Porous Media on the Creeping Flow through Round Vessel. J Appl Computat Math 7: 399. doi: 10.4172/2168-9679.1000399

Page 6 of 6

17. Siddiqui AM, Haroon T, Shahzad A (2016) Hydrodynamics of viscous fluid through porous slit with linear absorption. App Math Mech 37: 361-378.

18. Siddiqui AM, Haroon T, Kahshan M, Iqbal MZ (2015) Slip Effects on the Flow of Newtonian Fluid in Renal Tubule. J Comput Theor Nanosci 12: 4319-4328.

19. Siddiqui AM, Sohail A, Naqvi S, Haroon T (2017) Analysis of Stokes flow through periodic permeable tubules. Alexandria Eng J 56: 105-113.

20. Ahmad S, Ahmad N (2011) On flow through renal tubule in case of periodic radial velocity component. International Journal of Emerging Multidisciplinary Fluid Sciences 3.

21. Berker R (1963) Integration of the equations of the motion of an incompressible viscous fluid. The Physics book 3: 1-384.

22. Bateman H (1932) Report of the Committee on Hydrodynamics. Bull Natl Research Council (US) 84: 634
23. Siddiqui AM, Zeb A, Ghori QK (2006) Some exact solution of 2D steady flow of an incompressible viscous fluid through porous medium. Journal of Porous Medium 9: 491-502.

24. Wang CY (1991) Exact solutions of the steady-state Navier-Stokes equations Annual Review of Fluid Mechanics 23: 159-177.

25. Siddiqui AM, Kaloni PN (1986) Certain inverse solutions of a non-Newtonian fluid. International Journal of Non-linear Mechanics 21: 459-473.

26. Siddiqui AM (1990) Some more inverse solutions of a non-Newtonian fluid Mech Res Commun 17: 157-163.

27. Zeb A, Siddiqui AM, Ahmed M (2013) An analysis of the flow of a Newtonian fluid between two moving parallel plates. ISRN Math Anal. 